OPEN ACCESS

Edited by:

José Luis Capelo,

Universidade Nova de Lisboa,

Portugal

Reviewed by:

Maria Soledad Ramirez,

California State University, Fullerton,

United States

M. Teresa Machini,

University of São Paulo, Brazil

*Correspondence:

Ayush Kumar

ayush.kumar@umanitoba.ca

Specialty section:

This article was submitted to

Antimicrobials, Resistance and

Chemotherapy

a section of the journal

Frontiers in Microbiology

Received: 22 August 2018 Accepted: 14 January 2019

Published: 30 January 2019

Citation:

De Silva PM and Kumar A (2019)

Signal Transduction Proteins in

Acinetobacter baumannii: Role in Antibiotic Resistance, Virulence, and

Potential as Drug Targets.

Front. Microbiol. 10:49

doi: 10.3389/fmicb.2019.00049

\section{Signal Transduction Proteins in Acinetobacter baumannii: Role in Antibiotic Resistance, Virulence, and Potential as Drug Targets}

\author{
P. Malaka De Silva ${ }^{1}$ and Ayush Kumar ${ }^{1,2 *}$ \\ ${ }^{1}$ Department of Microbiology, University of Manitoba, Winnipeg, MB, Canada, ${ }^{2}$ Manitoba Chemosensory Biology Group, \\ University of Manitoba, Winnipeg, MB, Canada
}

Acinetobacter baumannii is a notorious pathogen in health care settings around the world, primarily due to high resistance to antibiotics. A. baumannii also shows an impressive capability to adapt to harsh conditions in clinical settings, which contributes to its persistence in such conditions. Following their traditional role, the Two Component Systems (TCSs) present in A. baumannii play a crucial role in sensing and adapting to the changing environmental conditions. This provides $A$. baumannii with a greater chance of survival even in unfavorable conditions. Since all the TCSs characterized to date in A. baumannii play a role in its antibiotic resistance and virulence, understanding the underlying molecular mechanisms behind TCSs can help with a better understanding of the pathways that regulate these phenotypes. This can also guide efforts to target TCSs as novel drug targets. In this review, we discuss the roles of TCSs in A. baumannii, their molecular mechanisms, and most importantly, the potential of using small molecule inhibitors of TCSs as potential novel drug targets.

Keywords: two-component systems, PmrAB, AdeRS, BfmRS, stress

\section{INTRODUCTION}

Acinetobacter baumannii is a Gram-negative coccobacillus, which is an important opportunistic human pathogen that causes hospital-acquired infections (Peleg et al., 2008a, 2012; Visca et al., 2011; Wong et al., 2017). Clinical importance of A. baumannii is emphasized by the fact that it is listed by the WHO as the "top priority" pathogen that urgently need novel and effective therapeutic options (http://www.who.int/medicines/publications/WHOPPL-Short_Summary_25Feb-ET_NM_WHO.pdf). The success of A. baumannii in hospital environments can be mainly attributed to its ability to display multi-drug resistant phenotypes due to the rather robust acquisition of antibiotic resistance mechanisms (Dijkshoorn et al., 2007; Antunes et al., 2014). These include antibiotic modifying enzymes, decreased permeability to antibiotic molecules, and efflux pumps that extrude the antibiotic molecules out to the periplasm and beyond (Gordon and Wareham, 2010; Lee et al., 2017). 
Multi- and pan-drug resistance in A. baumannii is an alarming development for healthcare facilities around the world (Rodriguez-Bano et al., 2004; Agodi et al., 2010; Sievert et al., 2013; Labarca et al., 2016). As a result, some infections caused by multi-drug resistant $A$. baumannii have become virtually untreatable with our current arsenal of antibiotics (Maragakis and Perl, 2008). Further, without any new antibiotics for Gramnegative bacteria, such as A. baumannii in the developmental pipeline, we are on the verge of a post-antibiotic era where even a minor infection could have lethal consequences for the patient (Xie et al., 2018).

Apart from its multidrug resistance, the success of $A$. baumannii can also be attributed to its ability to survive and persist in the harsh conditions found within hospital environmental niches (Jawad et al., 1998; Rajamohan et al., 2010). Constant and prolonged exposure to antiseptics and desiccating agents, endurance of less than optimal temperatures, and sudden changes of the environmental and nutritional conditions when transferred into the human body from an abiotic surface are some of the challenges that A. baumannii faces in its role as an opportunistic human pathogen. Therefore, in order to be a successful pathogen, A. baumannii needs to sense and adapt to these changes in an efficient and timely manner.

Signal transduction mechanisms in bacteria play a crucial role in adapting to environmental changes. TCSs are one of the most ubiquitous signal transduction systems present in bacteria that help them sense and adapt to the environmental conditions (Alm et al., 2006; Wood et al., 2018). TCSs therefore play a role in bacterial adaptive responses which can lead to the modulation of their antibiotic susceptibility and virulence. Consequently, these systems are vital to study in order to understand the mechanisms of antibiotic resistance and virulence in bacteria (Poole, 2012; Kroger et al., 2016; Schaefers et al., 2017; Kenney, 2018; Lingzhi et al., 2018). Further, TCSs can also serve as an attractive target when developing anti-virulence therapeutics (Gotoh et al., 2010b). In this review, we describe the roles of TCSs in the resistance and virulence of A. baumannii and their potential to be used as novel therapeutic targets.

\section{TWO COMPONENT SYSTEMS (TCSS)}

TCSs are the most widespread signal transduction system present in bacteria and archaea (Stock et al., 2000). Typically, a TCS consists of two components, a histidine kinase (HK) and a response regulator (RR) (Figure 1). A high level of specificity with the HK and the RR is observed within the TCSs of a bacterial cell (Szurmant et al., 2007). However, there are instances where a single HK protein can have multiple cognate RR proteins (LopezRedondo et al., 2010) or when a single RR protein can be activated by multiple HK proteins (Laub and Goulian, 2007). Since their first description in 1986 (Nixon et al., 1986), an enormous amount of both HK and RR proteins have been discovered and characterized in a wide variety of bacteria (Whitworth and Cock, 2009). It is estimated that an average bacterial genome can contain up to 50-60 TCS-encoding genes (Whitworth, 2008; Whitworth and Cock, 2008; Wuichet et al., 2010). Given the advancement in bioinformatics and next generation sequencing techniques, specific databases dedicated to TCSs have become available that provide valuable information about these proteins (Ulrich and Zhulin, 2007; Barakat et al., 2011).

The TCSs in bacterial systems have implications for a wide variety of regulatory functions relating to sensing and adapting to their environment. In pathogenic bacteria, these functions often include but are not limited to antibiotic susceptibility modulation and virulence-related phenotypes, such as biofilm formation and motility (Tiwari et al., 2017).

\section{TCSs IN Acinetobacter baumannii}

An overview of various genomes of well-characterized $A$. baumannii clinical isolates show the presence of close to 20 different genes/operons that encode for TCSs (Table 1). Most of these genes and operons have a high degree of conservation at nucleotide level, indicating that they may be involved in the important functions. However, as mentioned above, the effector domains of $A$. baumannii $\mathrm{RR}$ proteins can be quite diverse which is shown in Figure 2. Below we describe the TCSs in $A$. baumannii that have been characterized to date.

\section{AdeRS}

AdeRS is the first characterized and also the most studied TCS in $A$. baumannii. It was first described in a clinical strain $A$. baumannii BM4454, when the inactivation of adeS resulted in an increased susceptibility to aminoglycosides due to the downregulation of the RND efflux pump AdeABC (Marchand et al., 2004) (Figure 3). Since it was first identified, a number of mutations in either adeR, adeS, or both have been shown to be directly responsible for the overexpression of the AdeABC pump (Ruzin et al., 2007; Yoon et al., 2013; Sun et al., 2016). Considering AdeRS system's role in the expression of AdeABC, it can be said that it plays a role in the susceptibility of $A$. baumannii to antibiotics that are substrates of the AdeABC pump. Further, the overexpression of AdeABC efflux pump has been associated with the decreased susceptibility to tigecycline observed in some clinical isolates of A. baumannii (Sun et al., 2014; Yuhan et al., 2016) thus implicating an indirect role of AdeRS in the susceptibility toward tigecycline. This is important since tigecycline is one of the last resort antibiotics for the treatment of multidrug resistant $A$. baumannii infections $(\mathrm{Ni}$ et al., 2016). However, there needs to be further investigations into this due to the possibility of involvement of other factors for the observed tigecycline susceptibility (Yoon et al., 2013).

Recent transcriptomics data suggest that the role of AdeRS extends well-beyond the expression of AdeABC efflux pump. A study in A. baumannii AYE showed that AdeRS controls the expression of almost 600 different genes (Richmond et al., 2016). Products of a number of these genes are believed to play a role in virulence, biofilm formation and multi drug efflux activity. However, deletion of $a d e B$ in the same strain resulted in similar phenotypes as deletion of adeRS. This suggests that at least some phenotypic changes observed upon the adeRS deletion may be a result of the decreased expression of the AdeABC efflux pump (Richmond et al., 2016). 


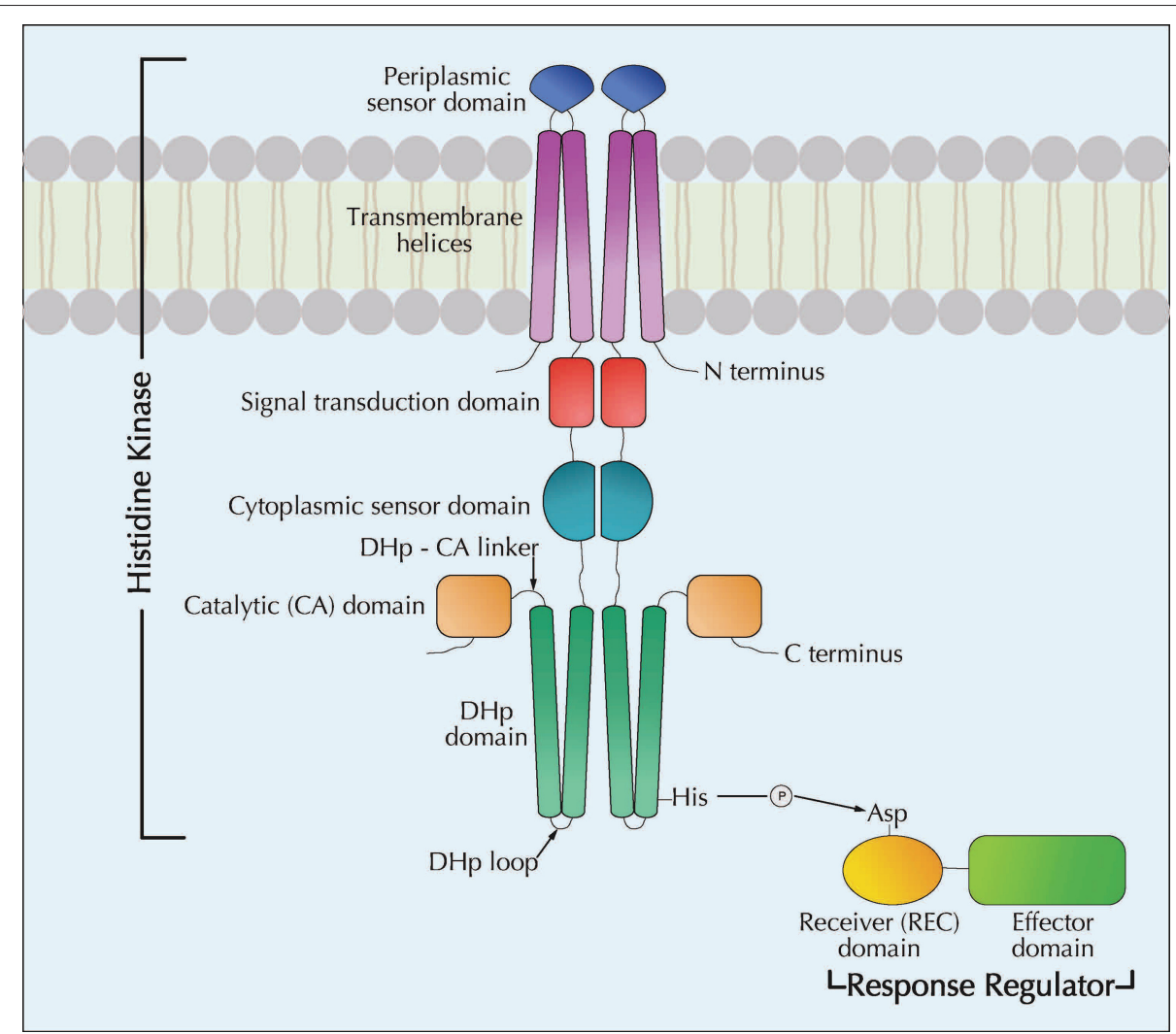

FIGURE 1 | Schematic diagram showing the cellular architecture of a typical two-component regulatory system as well the mechanism of phosphotransfer between two components (modified with permission from Springer Nature Du et al., 2018. A prototypical TCS, comprised of a membrane-bound sensory histidine kinase (HK) and a cytosolic response regulator (RR) protein, is shown. The basic mechanism of a TCS involves the HK sensing the environmental changes and relaying the message to the RR effectively through phosphorelays to initiate the necessary response. HK proteins, usually dimers, possess several conserved domains that are essential for their function, such as dimerization and histidine phosphotransfer (DHp) domain and catalytic ATP binding (CA) domain which make up the catalytic core of the HK (Bhate et al., 2015). The H-box containing the conserved histidine residue, that gets phosphorylated, is located in the DHp domain (Casino et al., 2009, 2010). The CA domain binds ATP and phosphorylates the histidine residue, thus initiating the HK autophosphorylation (Zschiedrich et al., 2016). The DHp and CA domains are conserved among all $\mathrm{HK}$ proteins and the sensory domains are variable conferring specificity of signal recognition. The phosphoryl group from the $\mathrm{H}$-box of the HK is ultimately transferred to a conserved aspartate residue of the receiver (REC) domain of the cognate RR thus activating the RR (Yamamoto et al., 2005). While the REC domain is highly conserved, the effector domains of RR display variability conferring specificity to the protein (Zschiedrich et al., 2016). Following its activation, dephosphorylation of the RR is critical to maintain the efficient regulatory capacity of the TCSs (Kenney, 2010). This is achieved through the phosphatase activity of the HK (Hsing and Silhavy, 1997).

The multifaceted regulon of AdeRS remains to be explored further, especially in clinically relevant phenotypes of $A$. baumannii. Further, environmental signals that activate the sensor kinase, AdeS, remain mostly unknown. However, we recently uncovered evidence that AdeRS system maybe responding to the $\mathrm{NaCl}$ concentrations in the growth medium (De Silva and Kumar, 2017). This work links adaption to environmental conditions, such as $\mathrm{NaCl}$ concentration to antibiotic susceptibility (as a result of expression of the AdeABC pump) as well as virulence factors, such as biofilm formation and surface-associated motility. It is therefore obvious that AdeRS plays a role in the antibiotic susceptibility of $A$. baumannii but also possibly in its virulence. However, it's role in antibiotic susceptibility and virulence is likely to be more strain-specific, as it is not uncommon to find disrupted copies of adeRS genes in clinical isolates of A. baumannii, such as LAC-4 and AB031 (Table 1).

\section{BaeSR}

BaeSR, named such because of its homology with an E. coli TCS (Leblanc et al., 2011), mediates a possible "cross-talk" with other TCSs. It has been shown to regulate overlapping regulons with other TCSs in A. baumannii. BaeSR was initially thought to be associated with the regulation of AdeABC RND efflux pump expression (Lin et al., 2014) (Figure 3). This is indicative of a possible cross-talk between BaeSR and AdeRS. Further investigations into the BaeSR revealed that it may also modulate the expression of AdeIJK and MacAB-TolC efflux pumps (Henry et al., 2012). However, efforts to determine the DNA binding sites in the promoters corresponding to the observed target genes remain unsuccessful, leaving room for further explorations (Lin et al., 2015). A phenotypic microarray screen revealed that the deletion of baeR resulted in reduced tolerance of $A$. baumannii to tannic acid (Lin et al., 2015), a diverse group of natural antibacterial compound (Henis et al., 1964). Tannic acids 


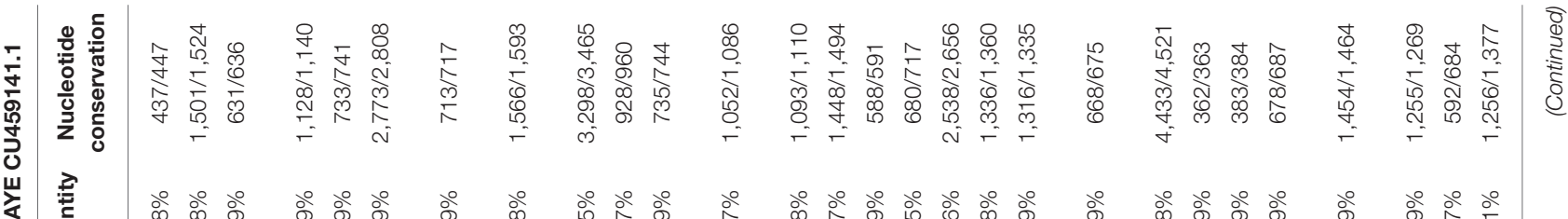

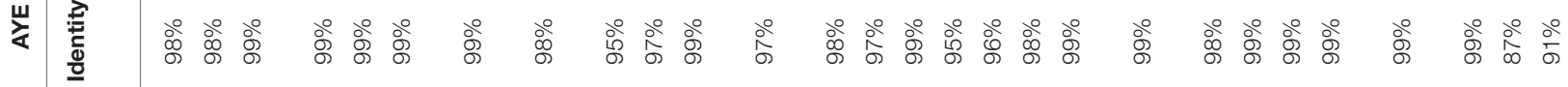

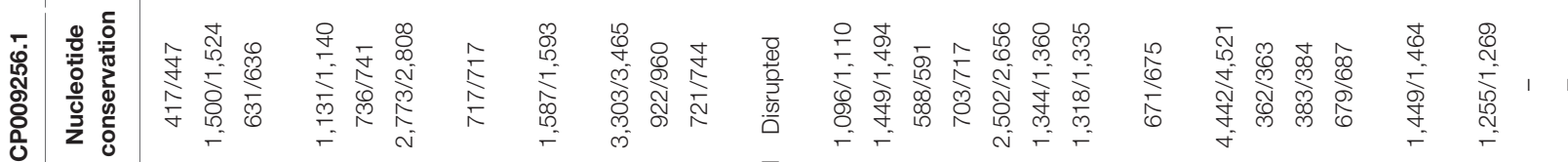

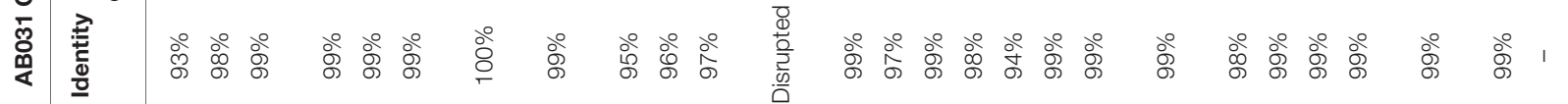

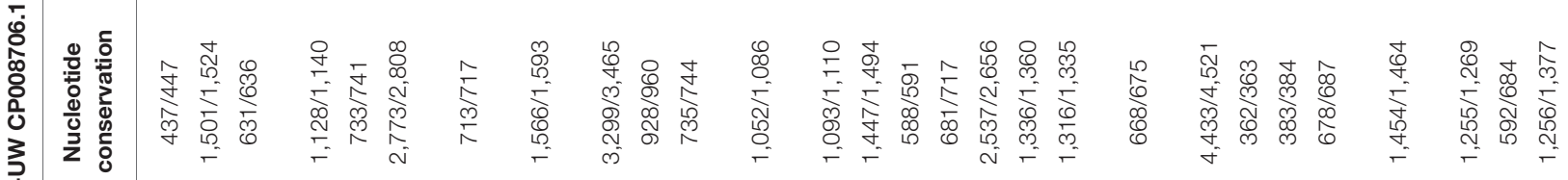

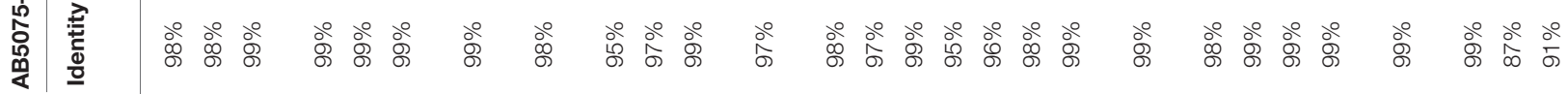

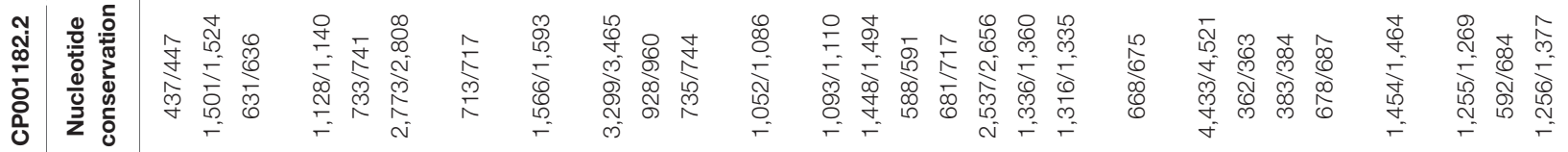

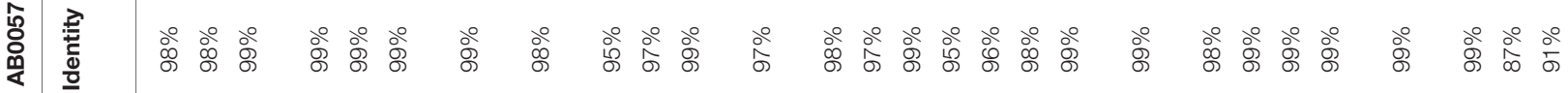

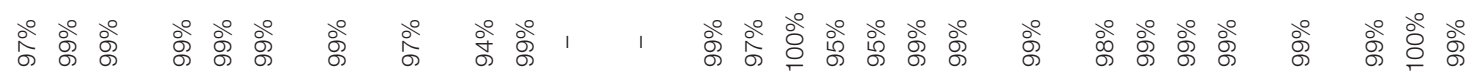

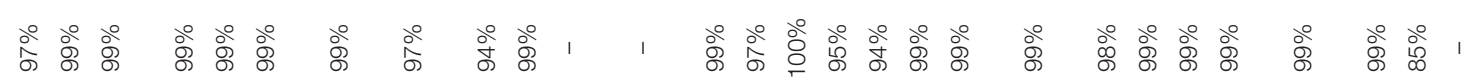

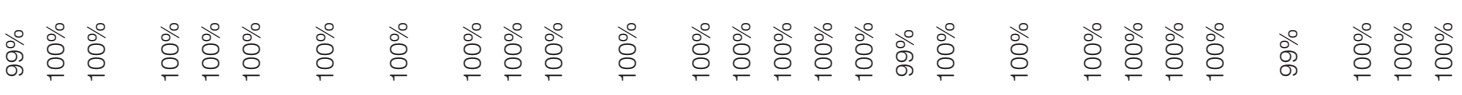




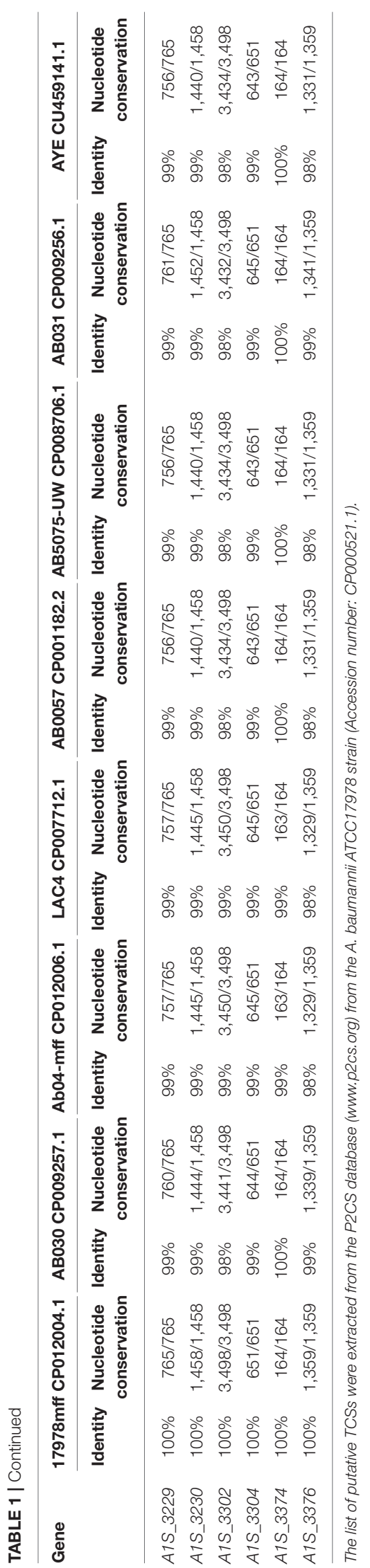

has been used as a topical agent in burn patients (Hupkens et al., 1995) as they display effective antibacterial activity against various bacteria, including E. coli, Staphylococcus aureus, Staphylococcus epidermidis, Salmonella spp. etc (Kim et al., 2016). Tannic acid has also been shown to inhibit biofilm formation in Staphylococcus aureus (Payne et al., 2013). In A. baumannii, Tannic acids are being explored as adjuvants for antimicrobial therapy. They were shown to synergize the activity of novobiocin, rifampicin, and fusidic acid against MDR A. baumannii (Chusri et al., 2009). However, the role of BaeSR TCS in tannic acid as well as the expression of efflux pumps controlled by BaeSR will have to be considered to in order to explore the clinical usage of tannic acid as an adjuvant therapy options against $A$. baumannii.

Studies on the environmental signals that BaeSR responds to remain limited. However, expression of baeR and baeS in A. baumannii is induced by sucrose $(20 \% \mathrm{w} / \mathrm{v}$ ) (Lin et al., 2014), suggesting that BaeSR may be involved in A. baumannii's response to osmotic stress.

\section{PmrAB}

A. baumannii's resistance to commonly used antibiotics has led to an increased use of "last resort" antibiotics, such as colistin (Karaiskos et al., 2017; Jiménez-Guerra et al., 2018). As a result, emergence of colistin resistance is becoming more common in $A$. baumannii (Cai et al., 2012; Lean et al., 2015). Investigations into the mechanisms of resistance to colistin in A. baumannii have revealed the involvement of PmrAB resistance (Park et al., 2011; Rolain et al., 2013), named so for its role in polymixin (Figure 3). PmrAB has been described in various Gram-negative pathogens including E. coli (Quesada et al., 2015), Salmonella enterica (Gunn, 2008), Klebsiella pneumoniae (Cheng et al., 2010), and Pseudomonas aeruginosa (Lee and Ko, 2014) and has been shown to have a similar function colistin resistance. Observations of mutations in both pmrA (RR) and $p m r B$ (HK) leading to decreased susceptibility to colistin presented preliminary evidence of the connection between PmrAB and colistin susceptibility in A. baumannii (Adams et al., 2009). Further, both colistin-resistant clinical isolates as well as laboratory generated spontaneous mutants showed phosphoethanolamine modification of lipid A of lipopolysaccharide (LPS) within the outer membrane (Arroyo et al., 2011; Beceiro et al., 2011). The modification of lipid A is mediated by $\mathrm{PmrC}$ which is generally part of the same operon as pmrAB (Raetz et al., 2007). PmrC can add phosphoethanolamine to either $4^{\prime}$ or $1^{\prime}$ phosphate of lipid A (Da Silva and Domingues, 2017). This modification of LPS results in a positively charged phosphate groups and prevents the binding of the cationic colistin (Tamayo et al., 2005a,b; Arroyo et al., 2011). Mutations in both $p m r A$ and $p m r B$ cause the overexpression of the pmrCAB operon.

Observations that low $\mathrm{pH}$ or supplementation of $\mathrm{Fe}^{3+}$ in the growth medium (Adams et al., 2009) lead to colistin resistance may suggest that $\mathrm{PmrB}$ could be responding to those signals (Gunn, 2008). However, growth of A. baumannii under low $\mathrm{pH}$ or in iron supplemented growth media failed to alter the expression of pmrA (Adams et al., 2009). Therefore, the environmental signals to which $\mathrm{PmrAB}$ responds to in $A$. baumannii remain elusive. 


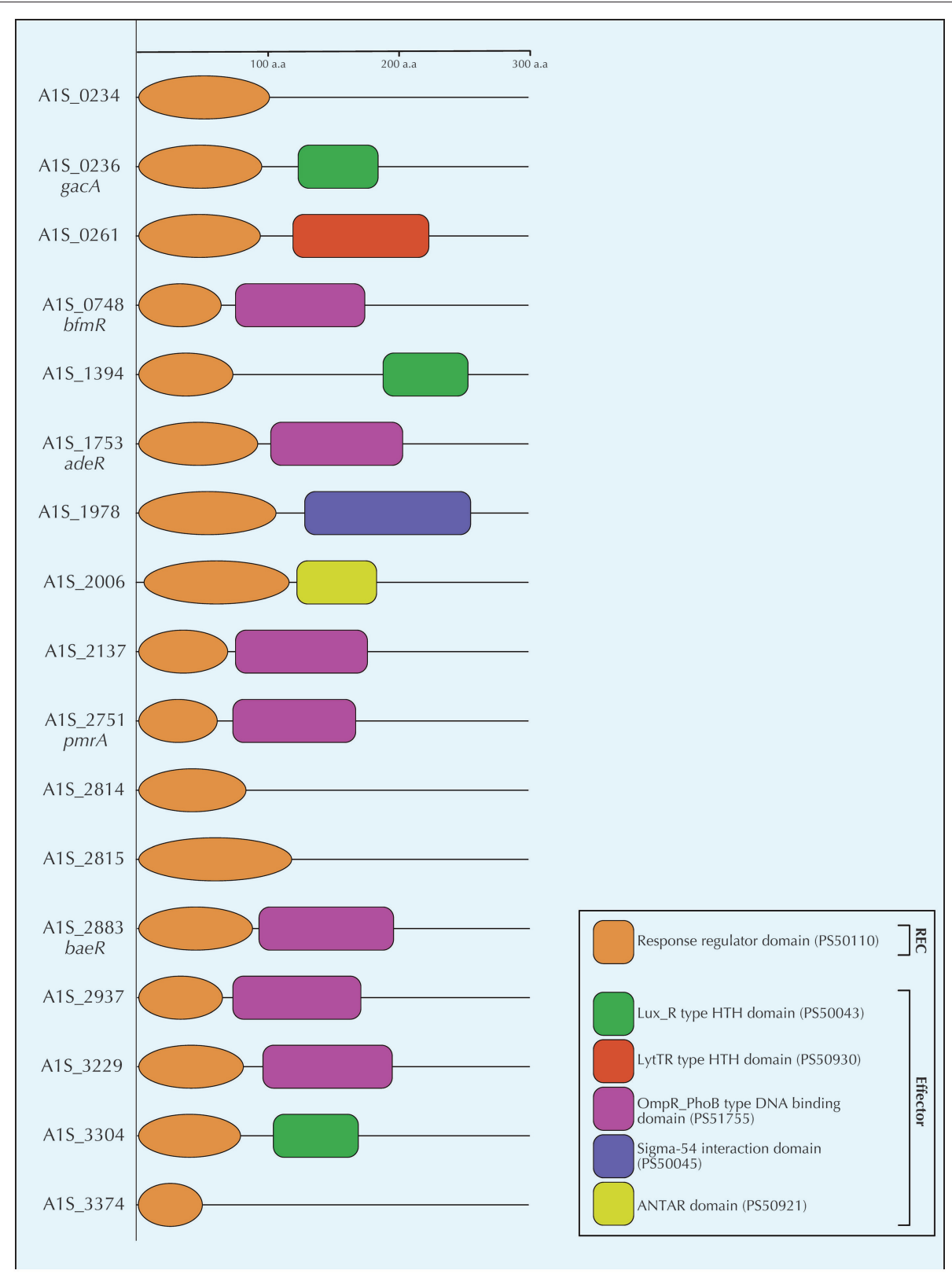

FIGURE 2 | Schematic diagram of the conserved domains of all the response regulators of A. baumannii ATCC17978 as determined by a ScanProsite (de Castro et al., 2006). The figure depicts the receiver domain (orange) and the different effector domains identified by ScanProsite (Lux_R type HTH domain in green, LytTR type HTH domain in red, OmpR_PhoB type DNA binding domain in violet, Sigma-54 interaction domain in purple, and ANTAR domain in yellow). Most abundant effector domain was the OmpR_PhoB type DNA binding domain which was present in seven response regulators followed by the Lux_R type HTH domain which was present in three response regulators. The other three types of effector domains were exclusive to single response regulators. The numbers in parenthesis refer to the PROSITE accession numbers of the respective domains. The hybrid sensor kinase A1S_2811 was not included in the figure due to the lack of a distinct response regulator protein.

\section{GacSA}

GacSA is a TCS that is well-characterized in Pseudomonas sp. (Gooderham and Hancock, 2009). GacSA in A. baumannii ATCC19606 was identified when the transposon insertions in the gacS sensor kinase gene rendered the mutants incapable of utilizing citrate as the sole carbon source (Dorsey et al., 2002). This suggests that GacSA is involved in citrate metabolism.
Since the initial characterization of GacSA in A. baumannii ATCC19606, a number of subsequent studies have carried out the functional characterization of GacSA TCS in A. baumannii ATCC17978. Interestingly, in A. baumannii ATCC17978, the gacS gene is not linked to the response regulator-encoding gene. Rather, it has both a HisKA domain and a REC domain suggesting that it could function as a hybrid sensor kinase. 


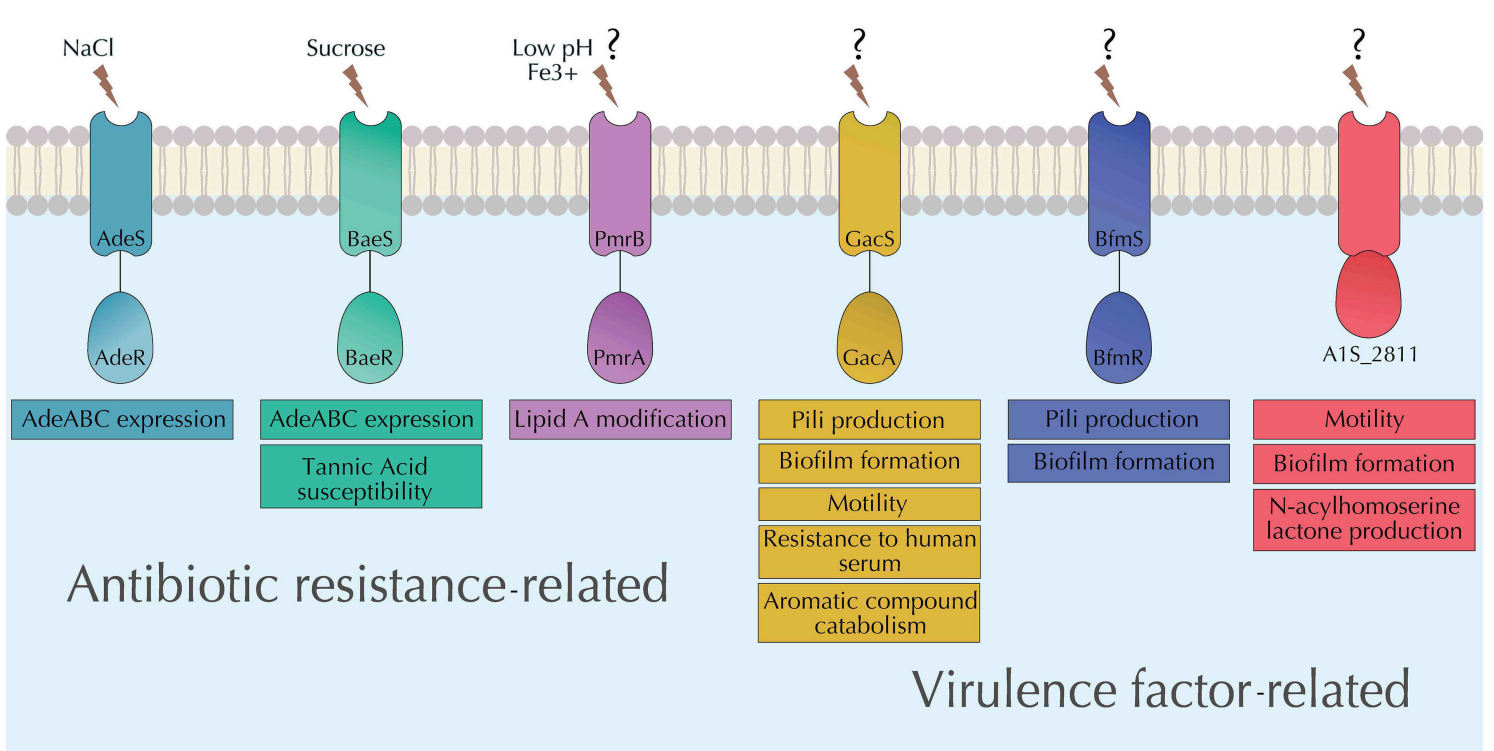

FIGURE 3 | Summary of the characterized TCSs in A. baumannii. Functions of each of the characterized TCSs in A. baumannii (AdeRS, BaeSR, PmrAB, GacSA, BfmRS, and A1S_2811) as well as their known stimuli are depicted.

Although, there is also a possibility that in A. baumannii ATCC17978, the response regulator for GacS is encoded elsewhere in the genome. This indicates that the organization of the gacSA genes may vary from strain to strain in A. baumannii.

In addition to the initially observed metabolic role of gacS, a transposon mutant with a disrupted gacS gene displayed significantly reduced $A$. baumannii's ability to inhibit Candida albicans (Peleg et al., 2008b). gacS deletion mutant also displayed attenuated virulence in a mouse infection model (Cerqueira et al., 2014). Deletion of gacS also led to the revelation of its involvement in a number of other virulence related functions. These include control of pili synthesis, motility, and biofilm formation, resistance against human serum, and metabolism of aromatic compounds (Cerqueira et al., 2014) (Figure 3). GacSA is involved in the regulation of the aromatic compound catabolism through the paa operon that encodes the components of the phenylacetic acid catabolic pathway. The paa gene cluster is significantly downregulated in the gacSA deletion mutants which may explain their attenuated virulence in a mouse septicaemia model (Cerqueira et al., 2014). The attenuated virulence of gacSA deletion mutants was observed in a later study involving a zebra fish virulence model as well (Bhuiyan et al., 2016) adding to the repertoire of studies that suggest that GacSA may function as a global virulence regulator in $A$. baumannii.

\section{BfmRS}

Biofilm formation is an important virulence factor of pathogens, such as A. baumannii that helps them survive harsh conditions present in hospital environments. The ability of $A$. baumannii to form biofilms starts with its attachment to surfaces that is mediated by the expression of pili. The expression of pili is mediated by the csu operon in A. baumannii and is under the regulatory control of BfmRS (Tomaras et al., 2008). Deletion of the response regulator $b f m R$ in A. baumannii ATCC19606 resulted in the complete abolishment of biofilm formation (Tomaras et al., 2008) (Figure 3). While of the role of $c s u$ operon in the attachment of A. baumannii on abiotic surfaces is wellestablished (Tomaras et al., 2003; Moon et al., 2017; Pakharukova et al., 2018), its role in the adherence of A. baumannii to human epithelial cells remains ambiguous. It was observed that A. baumannii ATCC19606 strain lacking csuE in fact adhered to bronchial epithelial cells better than the wild-type parent making the role pili in adherence to epithelial cells unclear (de Breij et al., 2009). It is possible that this was a strain specific outcome and further investigations are required to draw definitive conclusions.

In addition to regulating biofilm formation, BfmRS also plays a role in regulating the exopolysaccharide production (Geisinger et al., 2018). Exopolysaccharides play an important role in virulence of $A$. baumannii as they are a component of the capsule, which protects $A$. baumannii against serum killing and increasing the virulence in animal models. Further, antibiotic exposure leads to an increase in capsule production in A. baumannii mediated by increased expression of genes in K-locus, which in turn is regulated by the BfmRS system (Geisinger and Isberg, 2015).

Crystal structure of BfmR shows that it binds to its own promoter with higher affinity in an inactive (dephosphorylated) state compared to the active (phosphorylated) state (Draughn et al., 2018). This is unusual behavior highlights a unique selfregulation strategy of BfmRS system Therefore, BfmRS system is an excellent candidate to study not only the mechanisms that regulate virulence factors in A. baumannii but also the functioning of the TCSs systems in general. 


\section{A1S_2811}

A1S_2811 is a recently characterized hybrid sensor kinase possessing four histidine-containing phosphotransfer domains as well as a regulatory CheA-like domain and a CheYlike receiver domain (Chen et al., 2017). CheA and CheY homologs in $E$. coli and $P$. aeruginosa are associated with regulatory roles in controlling motility via regulating either pili or flagella (Li et al., 1995; Alon et al., 1998; Bertrand et al., 2010). Interestingly, in A. baumannii, this hybrid sensor kinase is expressed in an operon composed of five genes where the four other genes upstream are pilJ, pill, pilH, and pilG. Phenotypic analysis of the deletion mutant of A1S_2811 revealed a significant reduction in surface motility and biofilm formation at the gas-liquid interface. More intriguingly, abaI, which encodes a $\mathrm{N}$-acylhomoserine lactone involved in quorum sensing, was also significantly downregulated. Supplementation with synthetic homoserine lactone complemented the biofilm and motility phenotypes (Figure 3). This suggests that A1S_2811 regulates biofilm formation and surface motility through an AbaI-associated quorum sensing pathway rather than the conventional pili associated pathway (Chen et al., 2017). This is in contrast to the BfmRS mediated regulon controlling biofilm formation in A. baumannii. Association of both BfmRS and A1S_2811 with biofilm formation is also an example of one phenotype being under the control of multiple regulatory networks formed by different TCSs.

\section{TCSs AS POTENTIAL NOVEL DRUG TARGETS IN BACTERIAL PATHOGENS}

Given the important role that TCSs play in regulating the clinically-relevant phenotypes (virulence and/or antibiotic resistance) of bacterial pathogens, it has been proposed that targeting them therapeutically can offer an alternate treatment strategy against multidrug resistant pathogens (Barrett and Hoch, 1998; Stephenson and Hoch, 2002a,b, 2004; Gotoh et al., 2010b; Cardona et al., 2018). TCSs in A. baumannii as well as other organisms offer promise as novel drug targets because of a number of reasons; (i) their conserved nature among bacteria, (ii) their involvement in modulating antibiotic resistance and virulence phenotypes, (iii) their absence in mammalian cells thus reducing off-target toxicity, (iv) lesser potential of resistance development, as the focus of the approach is to supress virulence and/or antibiotic susceptibility rather than killing the cells. It is therefore not all that surprising that TCSs from different organisms have been studied as potential therapeutic targets. Table 2 summarizes a few examples of the use of TCSs inhibitors used in bacterial pathogens other than $A$. baumannii.

\section{POTENTIAL OF TCSS AS NOVEL DRUG TARGETS IN A. baumannii}

In A. baumannii, small molecule inhibitors, such as 2aminoimidazole compounds have shown great promise in inhibiting the action of both PmrA and BfmR. The 2aminoimidazole-based adjuvants used in combination with colistin were able to reverse colistin resistance in A. baumannii clinical isolates through inhibiting $\mathrm{PmrAB}$ and thereby abolishing the lipid A modification (Brackett et al., 2016). A promising feature of this strategy was that no resistance toward the PmrAB inhibitor was observed during the testing period of 7-days (Harris et al., 2014). Yet another example is the use of small molecule 2-aminoimidazole derivatives to inhibit the functions of BfmR (Thompson et al., 2012), such as biofilm formation (Milton et al., 2018). However, as with any other small molecule inhibitor, the cytotoxicity of the compounds used against $\mathrm{PmrAB}$ and BfmRS remains to be determined before the inhibitors could be deployed in a clinical setting.

Preliminary findings on the inhibition of BfmRS and PmrAB system are encouraging. In addition, AdeRS, A1S_2811, or GacSA can potentially be explored as therapeutic targets because of the important role they have been shown to play in the antibiotic resistance and virulence of $A$. baumannii.

\section{CHALLENGES IN TARGETING TCSs FOR THERAPUTICS}

Despite the fact that the investigations of the TCSs show an increasing amount of information being uncovered during the

TABLE 2 | A brief summary of the examples of using TCS inhibitors as a therapeutic option in bacterial pathogens other than A. baumannii.

\begin{tabular}{|c|c|c|c|c|}
\hline TCS & Organism(s) & Inhibitor & Inhibitory action & References \\
\hline AlgR1/AlgR2 & Pseudomonas aeruginosa & Thiazole derivatives & $\begin{array}{l}\text { Inhibition of AlgR1 phosphorylation and AlgR2 } \\
\text { kinase activity }\end{array}$ & Roychoudhury et al., 1993 \\
\hline & & $\begin{array}{l}\text { Walrycin A } \\
\text { Walrycin B }\end{array}$ & Inhibition of phosphotransfer from WalR & Gotoh et al., 2010a \\
\hline PhoPQ & Salmonella sp. & Radicicol & Activity against $\mathrm{PhoQ}$ & Guarnieri et al., 2008 \\
\hline VanSR & Enterococcus faecium & Thiazole derivatives & Inhibition of phosphotransfer to VanR & Ulijasz and Weisblum, 1999 \\
\hline
\end{tabular}


recent years, the majority of these efforts have focused on the cellular functions carried out by TCSs. This has left a void of information about the environmental signals that act as a trigger for the histidine kinase stimulation. The proposed stimuli for the already characterized TCSs are limited to osmotic stress for BaeSR (Lin et al., 2014), monovalent cations for AdeRS (De Silva and Kumar, 2017); and possibly low $\mathrm{pH}$ and $\mathrm{Fe}^{3+}$ for PmrAB (Gunn, 2008; Adams et al., 2009). Uncovering the environmental stimuli that activate a TCS response is critical in understanding the molecular pathways that are used for gene regulation by a particular TCS. These pathways can then be better exploited to render A. baumannii non-virulent and/or antibiotic susceptible. However, it is often difficult to determine these signals due to an array of practical reasons including, but not limited to, the potential ability of sensor kinases to detect multiple stimuli and difficulty in expressing, purifying, and experimenting with histidine kinase proteins in vitro in their natural conformations.

\section{CONCLUSIONS AND FUTURE PERSPECTIVES}

It is evident that the characterized TCSs present in A. baumannii are responsible for controlling a number of antibiotic resistance and virulence associated phenotypes, which contribute to the success of this organism as a human pathogen. Research on
TCSs in A. baumannii has extended our knowledge on virulence and resistance mechanisms in this organism over the last few years. However, there is still a considerable knowledge gap in comprehensive understanding of the complete TCS regulatory networks. Nonetheless, TCSs present themselves as potential targets for drug design and the use of 2-aminoimidazole compounds is are encouraging. A better characterization of these systems both genetically and functionally is key for the potential use of TCS as therapeutic targets.

\section{AUTHOR CONTRIBUTIONS}

All authors listed have made a substantial, direct and intellectual contribution to the work, and approved it for publication.

\section{ACKNOWLEDGMENTS}

AK's laboratory is supported by grants from the Natural Science and Engineering Council of Canada (NSERC, RGPIN-201505550), the Canadian Institutes of Health Research (CIHR, PJT152945), and Cystic Fibrosis Foundation (CFF). PD is supported by Graduate fellowships from the University of Manitoba. Authors thank Raelene Engelberg and Kaleigh Ducas-Mowchun for help with the editing of this manuscript. Authors dedicate this work to Asha, who died from XDR-A. baumannii infection.

\section{REFERENCES}

Adams, M. D., Nickel, G. C., Bajaksouzian, S., Lavender, H., Murthy, A. R., Jacobs, M. R., et al. (2009). Resistance to colistin in Acinetobacter baumannii associated with mutations in the PmrAB two-component system. Antimicrob. Agents Chemother. 53, 3628-3634. doi: 10.1128/AAC.00284-09

Agodi, A., Auxilia, F., Barchitta, M., Brusaferro, S., D’Alessandro, D., Montagna, M. T., et al. (2010). Building a benchmark through active surveillance of intensive care unit-acquired infections: the Italian network SPIN-UTI. J. Hosp. Infect. 74, 258-265. doi: 10.1016/j.jhin.2009.08.015

Alm, E., Huang, K., and Arkin, A. (2006). The evolution of two-component systems in bacteria reveals different strategies for niche adaptation. PLoS Comput. Biol. 2:e143. doi: 10.1371/journal.pcbi.0020143

Alon, U., Camarena, L., Surette, M. G., Aguera y Arcas, B., Liu, Y., Leibler, S., et al. (1998). Response regulator output in bacterial chemotaxis. EMBO J. 17, 4238-4248. doi: 10.1093/emboj/17.15.4238

Antunes, L. C., Visca, P., and Towner, K. J. (2014). Acinetobacter baumannii: evolution of a global pathogen. Pathog. Dis. 71, 292-301. doi: 10.1111/2049-632X.12125.

Arroyo, L. A., Herrera, C. M., Fernandez, L., Hankins, J. V., Trent, M. S., and Hancock, R. E. (2011). The pmrCAB operon mediates polymyxin resistance in Acinetobacter baumannii ATCC 17978 and clinical isolates through phosphoethanolamine modification of lipid A. Antimicrob. Agents Chemother. 55, 3743-3751. doi: 10.1128/AAC.00256-11

Barakat, M., Ortet, P., and Whitworth, D. E. (2011). P2CS: a database of prokaryotic two-component systems. Nucleic Acids Res. 39(Database issue), D771-D776. doi: 10.1093/nar/gkq1023

Barrett, J. F., and Hoch, J. A. (1998). Two-component signal transduction as a target for microbial anti-infective therapy. Antimicrob. Agents Chemother. 42, 1529-1536.

Beceiro, A., Llobet, E., Aranda, J., Bengoechea, J. A., Doumith, M., Hornsey, M., et al. (2011). Phosphoethanolamine modification of lipid A in colistin-resistant variants of Acinetobacter baumannii mediated by the

pmrAB two-component regulatory system. Antimicrob. Agents Chemother. 55, 3370-3379. doi: 10.1128/AAC.00079-11

Bertrand, J. J., West, J. T., and Engel, J. N. (2010). Genetic analysis of the regulation of type IV pilus function by the Chp chemosensory system of Pseudomonas aeruginosa. J. Bacteriol. 192, 994-1010. doi: 10.1128/JB.01390-09

Bhate, M. P., Molnar, K. S., Goulian, M., and DeGrado, W. F. (2015). Signal transduction in histidine kinases: insights from new structures. Structure 23, 981-994. doi: 10.1016/j.str.2015.04.002

Bhuiyan, M. S., Ellett, F., Murray, G. L., Kostoulias, X., Cerqueira, G. M., Schulze, K. E., et al. (2016). Acinetobacter baumannii phenylacetic acid metabolism influences infection outcome through a direct effect on neutrophil chemotaxis. Proc. Natl. Acad. Sci. U.S.A. 113, 9599-9604. doi: 10.1073/pnas.15231 16113

Brackett, C. M., Furlani, R. E., Anderson, R. G., Krishnamurthy, A., Melander, R. J., Moskowitz, S. M., et al. (2016). Second generation modifiers of colistin resistance show enhanced activity and lower inherent toxicity. Tetrahedron 72 , 3549-3553. doi: 10.1016/j.tet.2015.09.019

Cai, Y., Chai, D., Wang, R., Liang, B., and Bai, N. (2012). Colistin resistance of Acinetobacter baumannii: clinical reports, mechanisms and antimicrobial strategies. J. Antimicrob. Chemother. 67, 1607-1615. doi: 10.1093/jac/dks084

Cardona, S. T., Choy, M., and Hogan, A. M. (2018). Essential two-component systems regulating cell envelope functions: opportunities for novel antibiotic therapies. J. Membr. Biol. 251, 75-89. doi: 10.1007/s00232-017-9995-5

Casino, P., Rubio, V., and Marina, A. (2009). Structural insight into partner specificity and phosphoryl transfer in two-component signal transduction. Cell 139, 325-336. doi: 10.1016/j.cell.2009.08.032

Casino, P., Rubio, V., and Marina, A. (2010). The mechanism of signal transduction by two-component systems. Curr. Opin. Struct. Biol. 20, 763-771. doi: 10.1016/j.sbi.2010.09.010

Cerqueira, G. M., Kostoulias, X., Khoo, C., Aibinu, I., Qu, Y., Traven, A., et al. (2014). A global virulence regulator in Acinetobacter baumannii and its control of the phenylacetic acid catabolic pathway. J. Infect. Dis. 210, 46-55. doi: 10.1093/infdis/jiu024 
Chen, R., Lv, R., Xiao, L., Wang, M., Du, Z., Tan, Y., et al. (2017). A1S_2811, a CheA/Y-like hybrid two-component regulator from Acinetobacter baumannii ATCC17978, is involved in surface motility and biofilm formation in this bacterium. Microbiologyopen 6:e510. doi: 10.1002/mbo3.510

Cheng, H. Y., Chen, Y. F., and Peng, H. L. (2010). Molecular characterization of the PhoPQ-PmrD-PmrAB mediated pathway regulating polymyxin B resistance in Klebsiella pneumoniae CG43. J. Biomed. Sci. 17:60. doi: 10.1186/1423-0127-17-60

Chusri, S., Villanueva, I., Voravuthikunchai, S. P., and Davies, J. (2009). Enhancing antibiotic activity: a strategy to control Acinetobacter infections. J. Antimicrob. Chemother. 64, 1203-1211. doi: 10.1093/jac/dkp381

Da Silva, G. J., and Domingues, S. (2017). Interplay between colistin resistance, virulence and fitness in Acinetobacter baumannii. Antibiotics (Basel) 6:E28. doi: 10.3390/antibiotics6040028

de Breij, A., Gaddy, J., van der Meer, J., Koning, R., Koster, A., van den Broek, P., et al. (2009). CsuA/BABCDE-dependent pili are not involved in the adherence of Acinetobacter baumannii ATCC19606(T) to human airway epithelial cells and their inflammatory response. Res. Microbiol. 160, 213-218. doi: 10.1016/j.resmic.2009.01.002

de Castro, E., Sigrist, C. J., Gattiker, A., Bulliard, V., Langendijk-Genevaux, P. S., Gasteiger, E., et al. (2006). ScanProsite: detection of PROSITE signature matches and ProRule-associated functional and structural residues in proteins. Nucleic Acids Res. 34(Web Server issue), W362-W365. doi: 10.1093/nar/gkl124

De Silva, P. M., and Kumar, A. (2017). Effect of sodium chloride on surfaceassociated motility of Acinetobacter baumannii and the role of AdeRS twocomponent system. J. Membr. Biol. 251, 5-13. doi: 10.1007/s00232-017-9985-7

Dijkshoorn, L., Nemec, A., and Seifert, H. (2007). An increasing threat in hospitals: multidrug-resistant Acinetobacter baumannii. Nat. Rev. Microbiol. 5, 939-951. doi: $10.1038 / \mathrm{nrmicro} 1789$

Dorsey, C. W., Tomaras, A. P., and Actis, L. A. (2002). Genetic and phenotypic analysis of Acinetobacter baumannii insertion derivatives generated with a transposome system. Appl. Environ. Microbiol. 68, 6353-6360. doi: 10.1128/AEM.68.12.6353-6360.2002

Draughn, G. L., Milton, M. E., Feldmann, E. A., Bobay, B. G., Roth, B. M., Olson, A. L., et al. (2018). The structure of the biofilm-controlling response regulator BfmR from Acinetobacter baumannii reveals details of its DNAbinding mechanism. J. Mol. Biol. 430, 806-821. doi: 10.1016/j.jmb.2018.02.002

Du, D., Wang-Kan, X., Neuberger, A., van Veen, H. W., Pos, K. M., Piddock, L. J. V., et al. (2018). Multidrug efflux pumps: structure, function and regulation. Nat. Rev. Microbiol. 16, 523-539. doi: 10.1038/s41579-018-0048-6

Geisinger, E., and Isberg, R. R. (2015). Antibiotic modulation of capsular exopolysaccharide and virulence in Acinetobacter baumannii. PLoS Pathog. 11:e1004691. doi: 10.1371/journal.ppat.1004691

Geisinger, E., Mortman, N. J., Vargas-Cuebas, G., Tai, A. K., and Isberg, R. R. (2018). A global regulatory system links virulence and antibiotic resistance to envelope homeostasis in Acinetobacter baumannii. PLoS Pathog. 14:e1007030. doi: 10.1371/journal.ppat.1007030

Gooderham, W. J., and Hancock, R. E. (2009). Regulation of virulence and antibiotic resistance by two-component regulatory systems in Pseudomonas aeruginosa. FEMS Microbiol. Rev. 33, 279-294. doi: 10.1111/j.1574-6976.2008.00135.x

Gordon, N. C., and Wareham, D. W. (2010). Multidrug-resistant Acinetobacter baumannii: mechanisms of virulence and resistance. Int. J. Antimicrob. Agents 35, 219-226. doi: 10.1016/j.ijantimicag.2009.10.024

Gotoh, Y., Doi, A., Furuta, E., Dubrac, S., Ishizaki, Y., Okada, M., et al. (2010a). Novel antibacterial compounds specifically targeting the essential WalR response regulator. J. Antibiot. 63, 127-134. doi: 10.1038/ja.2010.4

Gotoh, Y., Eguchi, Y., Watanabe, T., Okamoto, S., Doi, A., and Utsumi, R. (2010b). Two-component signal transduction as potential drug targets in pathogenic bacteria. Curr. Opin. Microbiol. 13, 232-239. doi: 10.1016/j.mib.2010. 01.008

Guarnieri, M. T., Zhang, L., Shen, J., and Zhao, R. (2008). The Hsp90 inhibitor radicicol interacts with the ATP-binding pocket of bacterial sensor kinase PhoQ. J. Mol. Biol. 379, 82-93. doi: 10.1016/j.jmb.2008.03.036

Gunn, J. S. (2008). The Salmonella PmrAB regulon: lipopolysaccharide modifications, antimicrobial peptide resistance and more. Trends Microbiol. 16, 284-290. doi: 10.1016/j.tim.2008.03.007
Harris, T. L., Worthington, R. J., Hittle, L. E., Zurawski, D. V., Ernst, R. K., and Melander, C. (2014). Small molecule downregulation of PmrAB reverses lipid A modification and breaks colistin resistance. ACS Chem. Biol. 9, 122-127. doi: $10.1021 / \mathrm{cb} 400490 \mathrm{k}$

Henis, Y., Tagari, H., and Volcani, R. (1964). Effect of water extracts of carob pods, tannic acid, and their derivatives on the morphology and growth of microorganisms. Appl. Microbiol. 12, 204-209.

Henry, R., Vithanage, N., Harrison, P., Seemann, T., Coutts, S., Moffatt, J. H., et al. (2012). Colistin-resistant, lipopolysaccharide-deficient Acinetobacter baumannii responds to lipopolysaccharide loss through increased expression of genes involved in the synthesis and transport of lipoproteins, phospholipids, and poly-beta-1,6-N-acetylglucosamine. Antimicrob. Agents Chemother. 56, 59-69. doi: 10.1128/AAC.05191-11

Hsing, W., and Silhavy, T. J. (1997). Function of conserved histidine-243 in phosphatase activity of EnvZ, the sensor for porin osmoregulation in Escherichia coli. J. Bacteriol. 179, 3729-3735.

Hupkens, P., Boxma, H., and Dokter, J. (1995). Tannic acid as a topical agent in burns: historical considerations and implications for new developments. Burns 21, 57-61.

Igarashi, M., Watanabe, T., Hashida, T., Umekita, M., Hatano, M., Yanagida, Y., et al. (2013). Waldiomycin, a novel WalK-histidine kinase inhibitor from Streptomyces sp. MK844-mF10. J. Antibiot. 66, 459-464. doi: $10.1038 /$ ja.2013.33

Jawad, A., Seifert, H., Snelling, A. M., Heritage, J., and Hawkey, P. M. (1998). Survival of Acinetobacter baumannii on dry surfaces: comparison of outbreak and sporadic isolates. J. Clin. Microbiol. 36, 1938-1941.

Jiménez-Guerra, G., Heras-Cañas, V., Gutiérrez-Soto, M., del Pilar AznartePadial, M., Expósito-Ruiz, M., Navarro-Marí, J. M., et al. (2018). Urinary tract infection by Acinetobacter baumannii and Pseudomonas aeruginosa: evolution of antimicrobial resistance and therapeutic alternatives. J. Med. Microbiol. 67, 790-797. doi: 10.1099/jmm.0.000742

Karaiskos, I., Souli, M., Galani, I., and Giamarellou, H. (2017). Colistin: still a lifesaver for the 21st century? Expert Opin. Drug Metab. Toxicol. 13, 59-71. doi: 10.1080/17425255.2017.1230200

Kenney, L. J. (2010). How important is the phosphatase activity of sensor kinases? Curr. Opin. Microbiol. 13, 168-176. doi: 10.1016/j.mib.2010.01.013

Kenney, L. J. (2018). The role of acid stress in Salmonella pathogenesis. Curr. Opin. Microbiol. 47, 45-51. doi: 10.1016/j.mib.2018.11.006

Kim, T. Y., Cha, S.-H., Cho, S., and Park, Y. (2016). Tannic acid-mediated green synthesis of antibacterial silver nanoparticles. Arch. Pharm. Res. 39, 465-473. doi: $10.1007 /$ s12272-016-0718-8

Kroger, C., Kary, S. C., Schauer, K., and Cameron, A. D. (2016). Genetic regulation of virulence and antibiotic resistance in Acinetobacter baumannii. Genes (Basel) 8:12. doi: $10.3390 /$ genes 8010012

Labarca, J. A., Salles, M. J., Seas, C., and Guzman-Blanco, M. (2016). Carbapenem resistance in Pseudomonas aeruginosa and Acinetobacter baumannii in the nosocomial setting in Latin America. Crit. Rev. Microbiol. 42, 276-292. doi: 10.3109/1040841X.2014.940494

Laub, M. T., and Goulian, M. (2007). Specificity in two-component signal transduction pathways. Annu. Rev. Genet. 41, 121-145. doi: 10.1146/annurev.genet.41.042007.170548

Lean, S. S., Yeo, C. C., Suhaili, Z., and Thong, K. L. (2015). Comparative genomics of two ST 195 carbapenem-resistant Acinetobacter baumannii with different susceptibility to polymyxin revealed underlying resistance mechanism. Front. Microbiol. 6:1445. doi: 10.3389/fmicb.2015.01445

Leblanc, S. K., Oates, C. W., and Raivio, T. L. (2011). Characterization of the induction and cellular role of the BaeSR two-component envelope stress response of Escherichia coli. J. Bacteriol. 193, 3367-3375. doi: 10.1128/JB.01534-10

Lee, C. R., Lee, J. H., Park, M., Park, K. S., Bae, I. K., Kim, Y. B., et al. (2017). Biology of Acinetobacter baumannii: pathogenesis, antibiotic resistance mechanisms, and prospective treatment options. Front. Cell. Infect. Microbiol. 7:55. doi: $10.3389 /$ fcimb. 2017.00055

Lee, J. Y., and Ko, K. S. (2014). Mutations and expression of PmrAB and PhoPQ related with colistin resistance in Pseudomonas aeruginosa clinical isolates. Diagn. Microbiol. Infect. Dis. 78, 271-276. doi: 10.1016/j.diagmicrobio.2013.11.027 
Li, J., Swanson, R. V., Simon, M. I., and Weis, R. M. (1995). The response regulators $\mathrm{CheB}$ and $\mathrm{CheY}$ exhibit competitive binding to the kinase CheA. Biochemistry 34, 14626-14636.

Lin, M. F., Lin, Y. Y., and Lan, C. Y. (2015). The role of the twocomponent system BaeSR in disposing chemicals through regulating transporter systems in Acinetobacter baumannii. PLoS ONE 10:e0132843. doi: 10.1371/journal.pone. 0132843

Lin, M. F., Lin, Y. Y., Yeh, H. W., and Lan, C. Y. (2014). Role of the BaeSR two-component system in the regulation of Acinetobacter baumannii adeAB genes and its correlation with tigecycline susceptibility. BMC Microbiol. 14:119. doi: 10.1186/1471-2180-14-119

Lingzhi, L., Haojie, G., Dan, G., Hongmei, M., Yang, L., Mengdie, J., et al. (2018). The role of two-component regulatory system in beta-lactam antibiotics resistance. Microbiol. Res. 215, 126-129. doi: 10.1016/j.micres.2018.07.005

Lopez-Redondo, M. L., Moronta, F., Salinas, P., Espinosa, J., Cantos, R., Dixon, R., et al. (2010). Environmental control of phosphorylation pathways in a branched two-component system. Mol. Microbiol. 78, 475-489. doi: $10.1111 / j .1365-2958.2010 .07348 . x$

Maragakis, L. L., and Perl, T. M. (2008). Acinetobacter baumannii: epidemiology, antimicrobial resistance, and treatment options. Clin. Infect. Dis. 46, 1254-1263. doi: $10.1086 / 529198$

Marchand, I., Damier-Piolle, L., Courvalin, P., and Lambert, T. (2004). Expression of the RND-type efflux pump AdeABC in Acinetobacter baumannii is regulated by the AdeRS two-component system. Antimicrob. Agents Chemother. 48, 3298-3304. doi: 10.1128/AAC.48.9.3298-3304.2004

Milton, M. E., Minrovic, B. M., Harris, D. L., Kang, B., Jung, D., Lewis, C. P., et al. (2018). Re-sensitizing multidrug resistant bacteria to antibiotics by targeting bacterial response regulators: characterization and comparison of interactions between 2-aminoimidazoles and the response regulators $\mathrm{BfmR}$ from Acinetobacter baumannii and QseB from Francisella spp. Front. Mol. Biosci. 5:15. doi: 10.3389/fmolb.2018.00015

Moon, K. H., Weber, B. S., and Feldman, M. F. (2017). Subinhibitory concentrations of trimethoprim and sulfamethoxazole prevent biofilm formation by Acinetobacter baumannii through inhibition of Csu Pilus expression. Antimicrob. Agents Chemother. 61:e00778-17. doi: 10.1128/AAC.00778-17

Ni, W., Han, Y., Zhao, J., Wei, C., Cui, J., Wang, R., et al. (2016). Tigecycline treatment experience against multidrug-resistant Acinetobacter baumannii infections: a systematic review and meta-analysis. Int. J. Antimicrob. Agents 47, 107-116. doi: 10.1016/j.ijantimicag.2015.11.011

Nixon, B. T., Ronson, C. W., and Ausubel, F. M. (1986). Two-component regulatory systems responsive to environmental stimuli share strongly conserved domains with the nitrogen assimilation regulatory genes ntrB and ntrC. Proc. Natl. Acad. Sci. U.S.A. 83, 7850-7854.

Okada, A., Igarashi, M., Okajima, T., Kinoshita, N., Umekita, M., Sawa, R., et al. (2010). Walkmycin B targets WalK (YycG), a histidine kinase essential for bacterial cell growth. J. Antibiot. 63, 89-94. doi: 10.1038/ja.2009.128

Pakharukova, N., Tuittila, M., Paavilainen, S., Malmi, H., Parilova, O., Teneberg, S., et al. (2018). Structural basis for Acinetobacter baumannii biofilm formation. Proc. Natl. Acad. Sci. U.S.A. 115, 5558-5563. doi: 10.1073/pnas.1800961115

Park, Y. K., Choi, J. Y., Shin, D., and Ko, K. S. (2011). Correlation between overexpression and amino acid substitution of the PmrAB locus and colistin resistance in Acinetobacter baumannii. Int. J. Antimicrob. Agents 37, 525-530. doi: 10.1016/j.ijantimicag.2011.02.008

Payne, D. E., Martin, N. R., Parzych, K. R., Rickard, A. H., Underwood, A., and Boles, B. R. (2013). Tannic acid inhibits Staphylococcus aureus surface colonization in an IsaA-dependent manner. Infect. Immun. 81, 496-504. doi: 10.1128/iai.00877-12

Peleg, A. Y., de Breij, A., Adams, M. D., Cerqueira, G. M., Mocali, S., Galardini, M., et al. (2012). The success of acinetobacter species; genetic, metabolic and virulence attributes. PLoS ONE 7:e46984. doi: 10.1371/journal.pone.0046984

Peleg, A. Y., Seifert, H., and Paterson, D. L. (2008a). Acinetobacter baumannii: emergence of a successful pathogen. Clin. Microbiol. Rev. 21, 538-582. doi: 10.1128/CMR.00058-07

Peleg, A. Y., Tampakakis, E., Fuchs, B. B., Eliopoulos, G. M., Moellering, R. C. Jr., and Mylonakis, E. (2008b). Prokaryote-eukaryote interactions identified by using Caenorhabditis elegans. Proc. Natl. Acad. Sci. U.S.A. 105, 14585-14590. doi: 10.1073/pnas.0805048105
Poole, K. (2012). Bacterial stress responses as determinants of antimicrobial resistance. J. Antimicrob. Chemother. 67, 2069-2089. doi: 10.1093/jac/dks196

Quesada, A., Porrero, M. C., Tellez, S., Palomo, G., Garcia, M., and Dominguez, L. (2015). Polymorphism of genes encoding PmrAB in colistin-resistant strains of Escherichia coli and Salmonella enterica isolated from poultry and swine. J. Antimicrob. Chemother. 70, 71-74. doi: 10.1093/jac/dku320

Raetz, C. R., Reynolds, C. M., Trent, M. S., and Bishop, R. E. (2007). Lipid A modification systems in gram-negative bacteria. Annu. Rev. Biochem. 76, 295-329. doi: 10.1146/annurev.biochem.76.010307.145803

Rajamohan, G., Srinivasan, V. B., and Gebreyes, W. A. (2010). Molecular and functional characterization of a novel efflux pump, AmvA, mediating antimicrobial and disinfectant resistance in Acinetobacter baumannii. J. Antimicrob. Chemother. 65, 1919-1925. doi: 10.1093/jac/dkq195

Rasko, D. A., Moreira, C. G., Li de, R., Reading, N. C., Ritchie, J. M., Waldor, M. K., et al. (2008). Targeting QseC signaling and virulence for antibiotic development. Science 321, 1078-1080. doi: 10.1126/science.1160354

Richmond, G. E., Evans, L. P., Anderson, M. J., Wand, M. E., Bonney, L. C., Ivens, A., et al. (2016). The Acinetobacter baumannii two-component system AdeRS regulates genes required for multidrug efflux, biofilm formation, and virulence in a strain-specific manner. MBio 7, e00430-e00416. doi: 10.1128/mBio.00430-16

Rodriguez-Bano, J., Cisneros, J. M., Fernandez-Cuenca, F., Ribera, A., Vila, J., Pascual, A., et al. (2004). Clinical features and epidemiology of Acinetobacter baumannii colonization and infection in Spanish hospitals. Infect. Control Hosp. Epidemiol. 25, 819-824. doi: 10.1086/502302

Rolain, J. M., Diene, S. M., Kempf, M., Gimenez, G., Robert, C., and Raoult, D. (2013). Real-time sequencing to decipher the molecular mechanism of resistance of a clinical pan-drug-resistant Acinetobacter baumannii isolate from Marseille, France. Antimicrob. Agents Chemother. 57, 592-596. doi: 10.1128/AAC.01314-12

Roychoudhury, S., Zielinski, N. A., Ninfa, A. J., Allen, N. E., Jungheim, L. N., Nicas, T. I., et al. (1993). Inhibitors of two-component signal transduction systems: inhibition of alginate gene activation in Pseudomonas aeruginosa. Proc. Natl. Acad. Sci. U.S.A. 90, 965-969.

Ruzin, A., Keeney, D., and Bradford, P. A. (2007). AdeABC multidrug efflux pump is associated with decreased susceptibility to tigecycline in Acinetobacter calcoaceticus-Acinetobacter baumannii complex. J. Antimicrob. Chemother. 59, 1001-1004. doi: 10.1093/jac/dkm058

Schaefers, M. M., Liao, T. L., Boisvert, N. M., Roux, D., Yoder-Himes, D., and Priebe, G. P. (2017). An oxygen-sensing two-component system in the Burkholderia cepacia complex regulates biofilm, intracellular invasion, and pathogenicity. PLoS Pathog. 13:e1006116. doi: 10.1371/journal.ppat.1006116

Sievert, D. M., Ricks, P., Edwards, J. R., Schneider, A., Patel, J., Srinivasan, A., et al. (2013). Antimicrobial-resistant pathogens associated with healthcareassociated infections: summary of data reported to the National Healthcare Safety Network at the Centers for Disease Control and Prevention, 2009-2010. Infect. Control Hosp. Epidemiol. 34, 1-14. doi: 10.1086/668770

Stephenson, K., and Hoch, J. A. (2002a). Two-component and phosphorelay signal-transduction systems as therapeutic targets. Curr. Opin. Pharmacol. 2, 507-512. doi: 10.1016/S1471489202001947

Stephenson, K., and Hoch, J. A. (2002b). Virulence- and antibiotic resistanceassociated two-component signal transduction systems of Gram-positive pathogenic bacteria as targets for antimicrobial therapy. Pharmacol. Ther. 93, 293-305. doi: 10.1016/S0163-7258(02)00198-5

Stephenson, K., and Hoch, J. A. (2004). Developing inhibitors to selectively target two-component and phosphorelay signal transduction systems of pathogenic microorganisms. Curr. Med. Chem. 11, 765-773. doi: 10.2174/0929867043455765

Stock, A. M., Robinson, V. L., and Goudreau, P. N. (2000). Twocomponent signal transduction. Annu. Rev. Biochem. 69, 183-215. doi: 10.1146/annurev.biochem.69.1.183

Sun, J. R., Jeng, W. Y., Perng, C. L., Yang, Y. S., Soo, P. C., Chiang, Y. S., et al. (2016). Single amino acid substitution Gly186Val in AdeS restores tigecycline susceptibility of Acinetobacter baumannii. J. Antimicrob. Chemother. 71, 1488-1492. doi: 10.1093/jac/dkw002

Sun, J. R., Perng, C. L., Lin, J. C., Yang, Y. S., Chan, M. C., Chang, T. Y., et al. (2014). AdeRS combination codes differentiate the response to efflux pump inhibitors in tigecycline-resistant isolates of extensively drug-resistant 
Acinetobacter baumannii. Eur. J. Clin. Microbiol. Infect. Dis. 33, 2141-2147. doi: 10.1007/s10096-014-2179-7

Szurmant, H., White, R. A., and Hoch, J. A. (2007). Sensor complexes regulating two-component signal transduction. Curr. Opin. Struct. Biol. 17, 706-715. doi: 10.1016/j.sbi.2007.08.019

Tamayo, R., Choudhury, B., Septer, A., Merighi, M., Carlson, R., and Gunn, J. S. (2005a). Identification of cptA, a PmrA-regulated locus required for phosphoethanolamine modification of the Salmonella enterica serovar typhimurium lipopolysaccharide core. J. Bacteriol. 187, 3391-3399. doi: 10.1128/JB.187.10.3391-3399.2005

Tamayo, R., Prouty, A. M., and Gunn, J. S. (2005b). Identification and functional analysis of Salmonella enterica serovar Typhimurium PmrA-regulated genes. FEMS Immunol. Med. Microbiol. 43, 249-258. doi: 10.1016/j.femsim.2004.08.007

Thompson, R. J., Bobay, B. G., Stowe, S. D., Olson, A. L., Peng, L., Su, Z., et al. (2012). Identification of BfmR, a response regulator involved in biofilm development, as a target for a 2-Aminoimidazole-based antibiofilm agent. Biochemistry 51, 9776-9778. doi: 10.1021/bi3015289

Tiwari, S., Jamal, S. B., Hassan, S. S., Carvalho, P., Almeida, S., Barh, D., et al. (2017). Two-component signal transduction systems of pathogenic bacteria as targets for antimicrobial therapy: an overview. Front. Microbiol. 8:1878. doi: 10.3389/fmicb.2017.01878

Tomaras, A. P., Dorsey, C. W., Edelmann, R. E., and Actis, L. A. (2003). Attachment to and biofilm formation on abiotic surfaces by Acinetobacter baumannii: involvement of a novel chaperone-usher pili assembly system. Microbiology 149(Pt 12), 3473-3484. doi: 10.1099/mic.0. 26541-0

Tomaras, A. P., Flagler, M. J., Dorsey, C. W., Gaddy, J. A., and Actis, L. A. (2008). Characterization of a two-component regulatory system from Acinetobacter baumannii that controls biofilm formation and cellular morphology. Microbiology 154(Pt 11), 3398-3409. doi: 10.1099/mic.0.2008/019471-0

Ulijasz, A. T., and Weisblum, B. (1999). Dissecting the VanRS signal transduction pathway with specific inhibitors. J. Bacteriol. 181, 627-631.

Ulrich, L. E., and Zhulin, I. B. (2007). MiST: a microbial signal transduction database. Nucleic Acids Res. 35(Database issue), D386-D390. doi: $10.1093 / \mathrm{nar} / \mathrm{gkl} 1932$

Visca, P., Seifert, H., and Towner, K. J. (2011). Acinetobacter infectionan emerging threat to human health. IUBMB Life 63, 1048-1054. doi: 10.1002/iub.534

Whitworth, D. E. (2008). Genomes and knowledge-a questionable relationship? Trends Microbiol. 16, 512-519. doi: 10.1016/j.tim.2008.08.001

Whitworth, D. E., and Cock, P. J. (2008). Two-component systems of the myxobacteria: structure, diversity and evolutionary relationships. Microbiology 154(Pt 2), 360-372. doi: 10.1099/mic.0.2007/013672-0
Whitworth, D. E., and Cock, P. J. (2009). Evolution of prokaryotic two-component systems: insights from comparative genomics. Amino Acids 37, 459-466. doi: 10.1007/s00726-009-0259-2

Wong, D., Nielsen, T. B., Bonomo, R. A., Pantapalangkoor, P., Luna, B., and Spellberg, B. (2017). Clinical and pathophysiological overview of acinetobacter infections: a century of challenges. Clin. Microbiol. Rev. 30, 409-447. doi: 10.1128/CMR.00058-16

Wood, C. R., Mack, L. E., and Actis, L. A. (2018). An update on the Acinetobacter baumannii regulatory circuitry. Trends Microbiol. 26, 560-562. doi: 10.1016/j.tim.2018.05.005

Wuichet, K., Cantwell, B. J., and Zhulin, I. B. (2010). Evolution and phyletic distribution of two-component signal transduction systems. Curr. Opin. Microbiol. 13, 219-225. doi: 10.1016/j.mib.2009.12.011

Xie, R., Zhang, X. D., Zhao, Q., Peng, B., and Zheng, J. (2018). Analysis of global prevalence of antibiotic resistance in Acinetobacter baumannii infections disclosed a faster increase in OECD countries. Emerg. Microbes Infect. 7:31. doi: 10.1038/s41426-018-0038-9

Yamamoto, K., Hirao, K., Oshima, T., Aiba, H., Utsumi, R., and Ishihama, A. (2005). Functional characterization in vitro of all two-component signal transduction systems from Escherichia coli. J. Biol. Chem. 280, 1448-1456. doi: $10.1074 /$ jbc.M410104200

Yoon, E. J., Courvalin, P., and Grillot-Courvalin, C. (2013). RND-type efflux pumps in multidrug-resistant clinical isolates of Acinetobacter baumannii: major role for AdeABC overexpression and AdeRS mutations. Antimicrob. Agents Chemother. 57, 2989-2995. doi: 10.1128/AAC.02556-12

Yuhan, Y., Ziyun, Y., Yongbo, Z., Fuqiang, L., and Qinghua, Z. (2016). Over expression of AdeABC and AcrAB-TolC efflux systems confers tigecycline resistance in clinical isolates of Acinetobacter baumannii and Klebsiella pneumoniae. Rev. Soc. Bras. Med. Trop. 49, 165-171. doi: 10.1590/0037-8682-0411-2015

Zschiedrich, C. P., Keidel, V., and Szurmant, H. (2016). Molecular mechanisms of two-component signal transduction. J. Mol. Biol. 428, 3752-3775. doi: 10.1016/j.jmb.2016.08.003

Conflict of Interest Statement: The authors declare that the research was conducted in the absence of any commercial or financial relationships that could be construed as a potential conflict of interest.

Copyright (๑) 2019 De Silva and Kumar. This is an open-access article distributed under the terms of the Creative Commons Attribution License (CC BY). The use, distribution or reproduction in other forums is permitted, provided the original author(s) and the copyright owner(s) are credited and that the original publication in this journal is cited, in accordance with accepted academic practice. No use, distribution or reproduction is permitted which does not comply with these terms. 